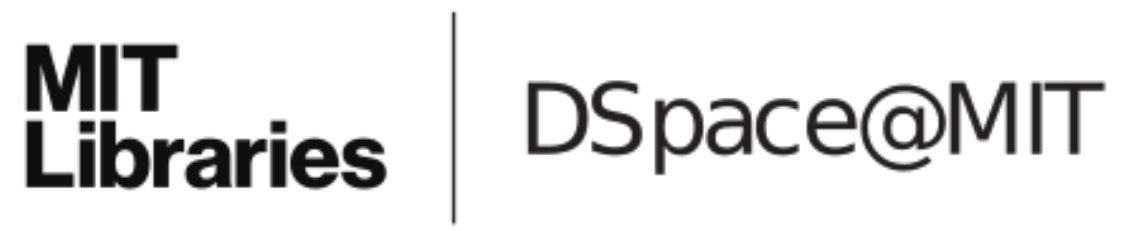

\author{
MIT Open Access Articles
}

Thermal nucleation and high-resolution imaging of submicrometer magnetic bubbles in thin thulium iron garnet films with perpendicular anisotropy

The MIT Faculty has made this article openly available. Please share how this access benefits you. Your story matters.

Citation: Büttner, Felix, et al. "Thermal nucleation and high-resolution imaging of submicrometer magnetic bubbles in thin thulium iron garnet films with perpendicular anisotropy." Physical Review Materials, 4, 1 (January 2020): 011401(R). (C 2020 American Physical Society

As Published: http://dx.doi.org/10.1103/PhysRevMaterials.4.011401

Publisher: American Physical Society (APS)

Persistent URL: https://hdl.handle.net/1721.1/125582

Version: Final published version: final published article, as it appeared in a journal, conference proceedings, or other formally published context

Terms of Use: Article is made available in accordance with the publisher's policy and may be subject to US copyright law. Please refer to the publisher's site for terms of use. 


\title{
Thermal nucleation and high-resolution imaging of submicrometer magnetic bubbles in thin thulium iron garnet films with perpendicular anisotropy
}

\author{
Felix Büttner $\odot,{ }^{1,},{ }^{*}$ Mohamad A. Mawass $\odot{ }^{2}$ Jackson Bauer $\odot,{ }^{1}$ Ethan Rosenberg, ${ }^{1}$ Lucas Caretta,,${ }^{1, \dagger}$ Can Onur Avci $\odot,{ }^{1, *}$ \\ Joachim Gräfe ${ }^{\circledR},{ }^{3}$ Simone Finizio $\odot,{ }^{4}$ C. A. F. Vaz $\odot,{ }^{4}$ Nina Novakovic $\odot,{ }^{2}$ Markus Weigand, ${ }^{2}$ Kai Litzius $\odot,{ }^{1}$ \\ Johannes Förster, ${ }^{3}$ Nick Träger, ${ }^{3}$ Felix Groß ${ }^{3}{ }^{3}$ Daniel Suzuki, ${ }^{1}$ Mantao Huang, ${ }^{1}$ Jason Bartell, ${ }^{1}$ Florian Kronast, ${ }^{2}$ \\ Jörg Raabe, ${ }^{4}$ Gisela Schütz, ${ }^{3}$ Caroline A. Ross $\odot,{ }^{1}$ and Geoffrey S. D. Beach ${ }^{1}$ \\ ${ }^{1}$ Department of Materials Science and Engineering, Massachusetts Institute of Technology, Cambridge, 02139 Massachusetts, USA \\ ${ }^{2}$ Helmholtz-Zentrum Berlin für Materialien und Energie, Albert-Einstein-Straße 15, 12489 Berlin, Germany \\ ${ }^{3}$ Max Planck Institute for Intelligent Systems, Heisenbergstraße 3, 70569 Stuttgart, Germany \\ ${ }^{4}$ Swiss Light Source, Paul Scherrer Institut, 5232 Villigen, Switzerland
}

(Received 5 November 2019; revised manuscript received 19 December 2019; published 28 January 2020)

\begin{abstract}
Ferrimagnetic iron garnets are promising materials for spintronics applications, characterized by ultralow damping and zero current shunting. It has recently been found that few nm-thick garnet films interfaced with a heavy metal can also exhibit sizable interfacial spin-orbit interactions, leading to the emergence, and efficient electrical control, of one-dimensional chiral domain walls. Two-dimensional bubbles, by contrast, have so far only been confirmed in micrometer-thick films. Here, we show by high resolution scanning transmission $\mathrm{x}$-ray microscopy and photoemission electron microscopy that submicrometer bubbles can be nucleated and stabilized in 25-nm-thick thulium iron garnet films via short heat pulses generated by electric current in an adjacent Pt strip, or by ultrafast laser illumination. We also find that quasistatic processes do not lead to the formation of a bubble state, suggesting that the thermodynamic path to reaching that state requires transient dynamics. X-ray imaging reveals that the bubbles have Bloch-type walls with random chirality and topology, indicating negligible chiral interactions at the garnet film thickness studied here. The robustness of thermal nucleation and the feasibility demonstrated here to image garnet-based devices by x-rays both in transmission geometry and with sensitivity to the domain wall chirality are critical steps to enabling the study of small spin textures and dynamics in perpendicularly magnetized thin-film garnets.
\end{abstract}

DOI: 10.1103/PhysRevMaterials.4.011401

\section{INTRODUCTION}

Iron garnets are insulating ferrimagnets with desirable properties in the context of magnetic soliton applications. Micrometer thick garnets were developed in the 1950s to 1980 s to realize the first commercial solid-state memory based on magnetic-field-driven magnetic bubbles [1-3]. This technology was ultimately not successful because propagating bubbles by magnetic fields is energetically expensive and not scalable. However, garnet materials have re-emerged as promising candidate materials for spintronics devices for many reasons: (i) they are electrically insulating, minimizing energy loss due to current shunting, (ii) they have low Gilbert damping, as low as $\sim 10^{-5}$ for YIG [4], which allows for long magnon diffusion lengths [5] and high domain wall mobilities [6,7], (iii) they exhibit a low depinning threshold $<4 \times 10^{10} \mathrm{~A} / \mathrm{m}^{2}$ to move domain walls electrically by spinorbit torques [6], and (iv) they are thermally and chemically more stable than metallic magnets.

\footnotetext{
*felixbuettner@gmail.com

†resent address: Department of Materials Science and Engineering, University of California, Berkeley, California 94720, USA.

${ }^{\ddagger}$ Present address: Department of Materials, ETH Zürich, 8093 Zürich, Switzerland.
}

The recent revival of garnet materials has been enabled by the successful growth of nanometer-thick, perpendicularly magnetized, epitaxial garnet films [8,9] with fundamentally different properties compared to bulk garnets. Most notably, thulium iron garnet $\left(\mathrm{TmIG}, \mathrm{Tm}_{3} \mathrm{Fe}_{5} \mathrm{O}_{12}\right)$ develops a significant chiral magnetic interaction (Dzyaloshinskii-Moriya interaction, DMI) at thicknesses of $\lesssim 6 \mathrm{~nm}[6,7]$. These fewunit-cell-thick garnet films can also be manipulated efficiently by pure spin currents generated in an adjacent heavy metal layer such as Pt $[6,7,10]$. Based on both ingredients, chirality and spin-torque, motion of domain wall spin textures with velocities exceeding $800 \mathrm{~m} / \mathrm{s}$ was recently observed in TmIG $[6,7]$. The existence of skyrmions has also been suggested recently by electrical signatures $[11,12]$ but remains to be confirmed by direct imaging.

High-resolution, in-operando $\mathrm{x}$-ray imaging has been a workhorse technique in skyrmion research [13-20]. The best resolution is achieved by transmission-based techniques, including scanning transmission $\mathrm{x}$-ray microscopy (STXM) [14,15,17-19] and x-ray holography [13,20], while photoemission electron microscopy (PEEM) can provide additional information about the Bloch or Néel character of domain walls [16] if the wall width is within the spatial resolution (typically $30 \mathrm{~nm}$ to $50 \mathrm{~nm}$ ). Key challenges to apply these techniques to garnets are the fabrication of membranes in case of transmission-based imaging, particularly without losing the 
(a)

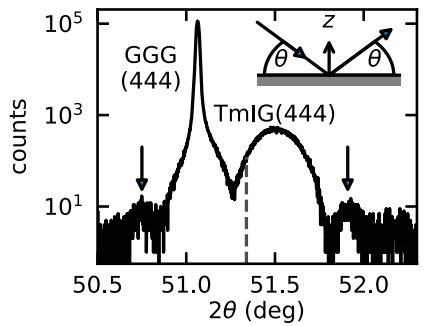

(b)

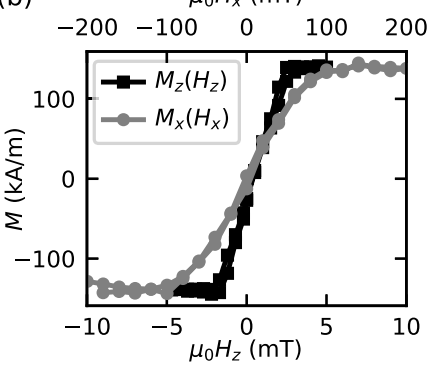

(a)

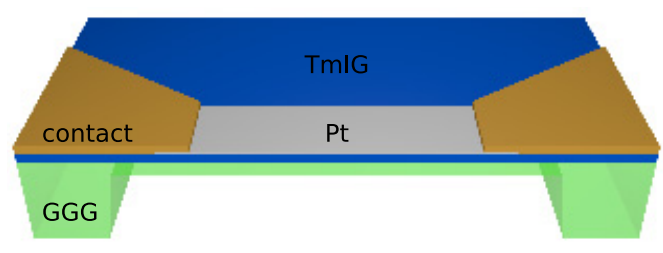

(b)
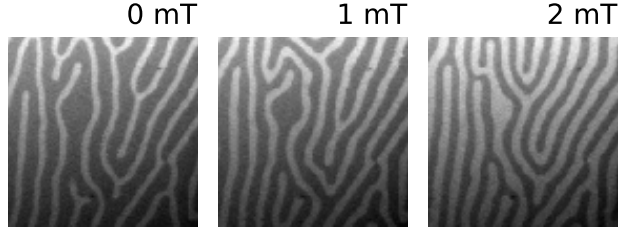

$3 \mathrm{mT}$

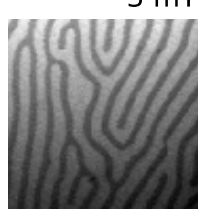

$4 \mathrm{mT}$
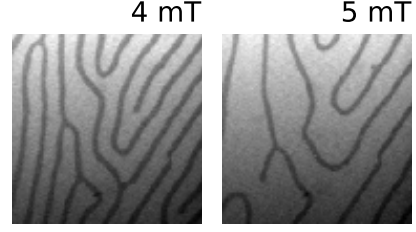

$6 \mathrm{mT}$

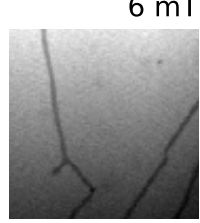

$7 \mathrm{mT}$
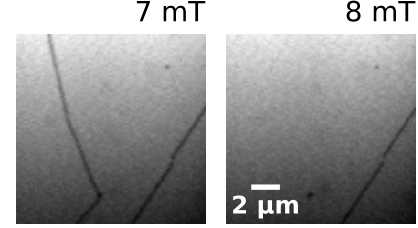

stable in a small bias field. This work not only demonstrates the thermally-induced formation and dynamics of bubbles in rare earth iron garnet films but also exemplifies the utility of $\mathrm{X}$-ray imaging in studying bubble and skyrmion behavior.

\section{RESULTS}

TmIG films with thicknesses of $26.5 \mathrm{~nm}$ and $30 \mathrm{~nm}(\sim 22$ and $\sim 25$ unit cells) were grown by pulsed laser deposition on (111)-oriented gadolinium gallium garnet (GGG) substrates $[8,9]$, see Methods. Symmetric $\theta-2 \theta$ x-ray diffraction scans [Fig. 1(a)] exhibit Laue fringes, confirming high crystalline quality, and show a shifted TmIG(444) peak corresponding to an out-of-plane $d_{444}$ spacing of $0.1773 \mathrm{~nm}$, compared to $0.178 \mathrm{~nm}$ (cubic lattice parameter $1.232 \mathrm{~nm}$ ) for bulk TmIG [21]. In-plane lattice matching to the substrate was confirmed by reciprocal space mapping. These results indicate pseudomorphic growth with in-plane tensile strain [8]. Combined with its negative magnetostriction coefficient $\lambda_{111}$, this produces a magnetoelastic anisotropy contribution favoring an out-of-plane easy axis in (111) TmIG [8,9]. Vibrating sample magnetometry was used to characterize the magnetic properties of the continuous films, as shown in Fig. 1(b). The saturation magnetization is $M_{s} \approx 140 \mathrm{kA} / \mathrm{m}$, slightly larger than the bulk value of $110 \mathrm{kA} / \mathrm{m}$. The out-of-plane saturation field of $\sim 2.5 \mathrm{mT}$ is much smaller than the in-plane saturation field of $\sim 100 \mathrm{mT}$, which is consistent with an out-of-plane easy axis with a demagnetized (multidomain) remanent state. This contrasts with the high-remanence out-of-plane loops for thinner TmIG films [8,9], pointing to stronger stray field interactions in these thicker films that promote a demagnetized state. The out-of-plane loop exhibits hysteresis near saturation, suggesting the presence of metastable states which can be transformed into a bubble ground state [22-24]. Finally, the small out-of-plane remanence and coercivity $<1 \mathrm{mT}$ suggest that the films have very low pinning.

FIG. 2. Properties of TmIG (30 nm) on a back-polished GGG membrane substrate. (a) Membrane device geometry for scanning transmission X-ray microscopy (STXM). (b) STXM images of the domain states of a bare $30 \mathrm{~nm}$ thick garnet film (without Pt layer) at increasing out-of-plane magnetic field. The contrast indicates the out-of-plane magnetization. Field values are shown on the top right of each image. The field was applied by rotating permanent magnets. Field values and the out-of-plane field direction are only approximate.

The domain configuration was imaged directly by high resolution scanning transmission $\mathrm{x}$-ray microscopy (STXM), with normal $\mathrm{x}$-ray incidence such that the $\mathrm{x}$-ray magnetic circular dichroism (XMCD) [25] contrast is sensitive to the out-of-plane magnetization direction. To achieve soft-x-ray transparency, the GGG substrates were mechanically polished to a thickness of $\sim 20 \mu \mathrm{m}$, and then a $\sim 40 \mu \mathrm{m} \times 40 \mu \mathrm{m}$ transmission window with $<1 \mu \mathrm{m}$ thickness was prepared using focused ion beam milling [see Methods and Fig. 2(a)]. We note that the Ga ion implantation depth is less than the final GGG membrane thickness, so Ga implantation in the magnetic film itself is not expected.

Near zero external field, the film exhibits a labyrinth multidomain remanent state with a high degree of alignment of the stripelike domains, as seen in Fig. 2(b). Hence, we conclude that strain relaxation during the milling process is minimal and the out-of-plane easy axis is retained. With increasing out-of-plane field $B_{z}$, the domains oriented parallel to the field grow in width, while the width and density of the antiparallel domains decrease as the film approaches saturation. (Note that the field was applied via rotating permanent magnets with $>200 \mathrm{mT}$ saturation field, possibly resulting in in-plane and out-of-plane field offsets of a few $\mathrm{mT}$, which may have been 


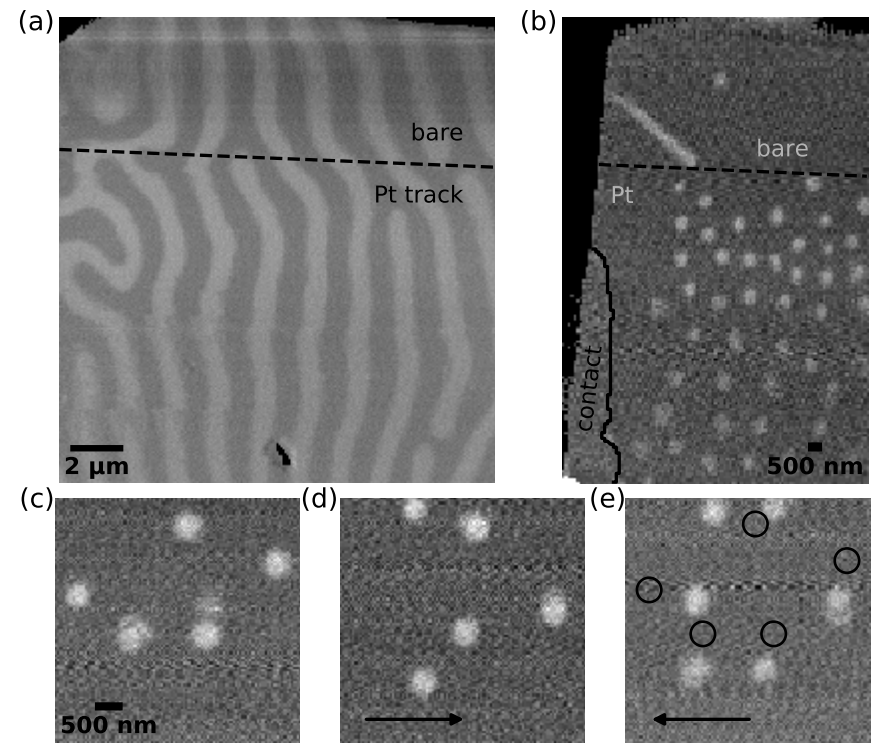

FIG. 3. Current-induced bubble nucleation and motion in $26.5 \mathrm{~nm}$ thick TmIG. (a) STXM image of a zero-field domain state in a TmIG film partly covered with a Pt track. The dashed line indicates the boundary of the Pt track. The image was taken immediately after inserting the sample into the instrument and the aligned orientation of the stripes (vertical in the top-view image) is possibly due to a previous exposure to an in-plane field. (b) STXM image after transmission of a single $100 \mathrm{~ns}$ current pulse of $8.2 \times 10^{11} \mathrm{~A} / \mathrm{m}^{2}$ amplitude in a pure out-of-plane field of $3.5 \mathrm{mT}$. (c)-(e) Images of bubble domains in (c) the initial state, (d) after application of a rightward-flowing current pulse, and (e) after subsequent application of a leftward-flowing current pulse, with $100 \mathrm{~ns}$ duration and $8.2 \times$ $10^{11} \mathrm{~A} / \mathrm{m}^{2}$ amplitude in an out-of-plane field of $4.5 \mathrm{mT}$. Solid circles show initial positions of the bubbles, from (c).

responsible for the preferred in-plane orientation of the stripe domains). Bubble domains are not observed here, which is not surprising, since at zero field, the parallel stripe phase is lower in energy than the bubble domain phase [23,24]. Transformation to a bubble phase would require overcoming sizable energy barriers, which is not expected during a quasistatic (adiabatic) increase in the applied field [23,24]. At the highest applied fields, the strip-out transition [26] is expected to lead to isolated bubbles formed from collapsed stripe domains, but their density in the present case is low enough that isolated bubbles are not observed within the STXM field of view.

Electrical current pulses have been recently used to nucleate magnetic skyrmions in metallic heavy-metal/ferro- and ferrimagnet heterostructures [20,24,27-29] and here we examine whether similar effects can be observed in magnetic insulators interfaced with a heavy metal. On top of a TmIG film, we patterned 4-nm-thick Pt tracks, $10 \mu \mathrm{m} \times 10 \mu \mathrm{m}$ in size, with 50-nm-thick $\mathrm{Pt}$ or $\mathrm{Au}$ contacts at either end for current injection [Fig. 2(a)] using lift-off processes prior to sample thinning. Figure 3(a) shows a STXM image at remanence of areas of the bare TmIG and an adjacent Ptcovered region. Stripe domains extend continuously across both regions. Some domains end at the edge of the Pt track, indicating pinning induced by the patterning process. However, there is no visible difference in the domain width. This suggests that the Pt overlay does not significantly contribute to the magnetic anisotropy (directly or through strain effects) or to an interfacial Dzyaloshinskii-Moriya interaction, both of which would change the equilibrium domain width [30].

While stripe domains are the lowest energy state at zero field, bubble states are favored by applied fields and eventually become the ground state of the system. We therefore increased the out-of-plane field to a value where all domains in our field of view disappeared $\left(B_{z}=3.5 \mathrm{mT}\right)$. At this field, we applied a unipolar current pulse (100 ns pulse duration; $8.2 \times 10^{11} \mathrm{~A} / \mathrm{m}^{2}$ amplitude). As shown in Fig. 3(b), this pulse nucleates a dense array of circular bubble domains, all of which have similar sizes of $\sim 500 \mathrm{~nm}$ in diameter. These bubbles appear almost exclusively under the Pt track, i.e., only where the current excitation was applied. There is a slight increase of the bubble density toward the Pt track edge, possibly due to the skin effect of the high frequency current. There are two possible explanations for the strong response of the magnetic material to current pulses in the $\mathrm{Pt}$ layer: (i) spin-orbit torques [31,32], which may arise from a pure spin current that is generated in the Pt layer due to the spin-Hall effect and (ii) thermal effects due to the Joule heating of the current pulse [24]. To distinguish between these mechanisms, we studied the response of nucleated bubbles to similar injected current pulses. Recent reports have shown that in few-unit-cells thin TmIG/Pt bilayers, the dampinglike torque from an injected spin Hall current can deterministically displace domain walls in the current-flow direction. This is enabled by a sizable Dzyaloshinskii-Moriya interaction that stabilizes Néel domain walls [6,7]. In the case of bubble domains, spin-orbit torques are expected to drive both Néel bubbles and Bloch bubbles, although the direction of motion would depend on the chirality and topology [33-35]. Even if the DMI is too weak to stabilize Néel domain walls in these relatively thick TmIG films, we expect that spin torques will drive each bubble in a deterministic and reversible manner.

Figures 3(c)-3(e) show sequential STXM images after positive and negative polarity current pulse injection of similar amplitude as before. Prior to this measurement, the bias field was increased to $4.5 \mathrm{mT}$ to reduce the density of bubbles to allow their tracking. We observe five bubble domains in all three frames [and a sixth bubble appearing at the top edge of Fig. 3(e)]. The approximately constant bubble count suggests that all three images show the same bubbles, only at different locations. However, the bubble displacement is random after each injected current pulse, and the displacement directions do not reverse when changing the polarity of the current pulse. The observations suggest that spin-orbit torques are not significant due to the relatively large thickness of our film and that Joule heating is dominantly responsible both for the nucleation and the motion of bubbles.

To confirm the role of thermal excitations in the observed bubble nucleation, we used ultrafast laser pulses to apply fast heat pulses in the absence of electrical excitations. Here, we used a nominally identical TmIG film on an unthinned GGG substrate. We applied 80 fs laser pulses (wavelength $800 \mathrm{~nm}$ ) of variable intensity and polarization through the polished backside of the sample (see Ref. [36] for details of the sample holder and optics). The resulting domain states were 

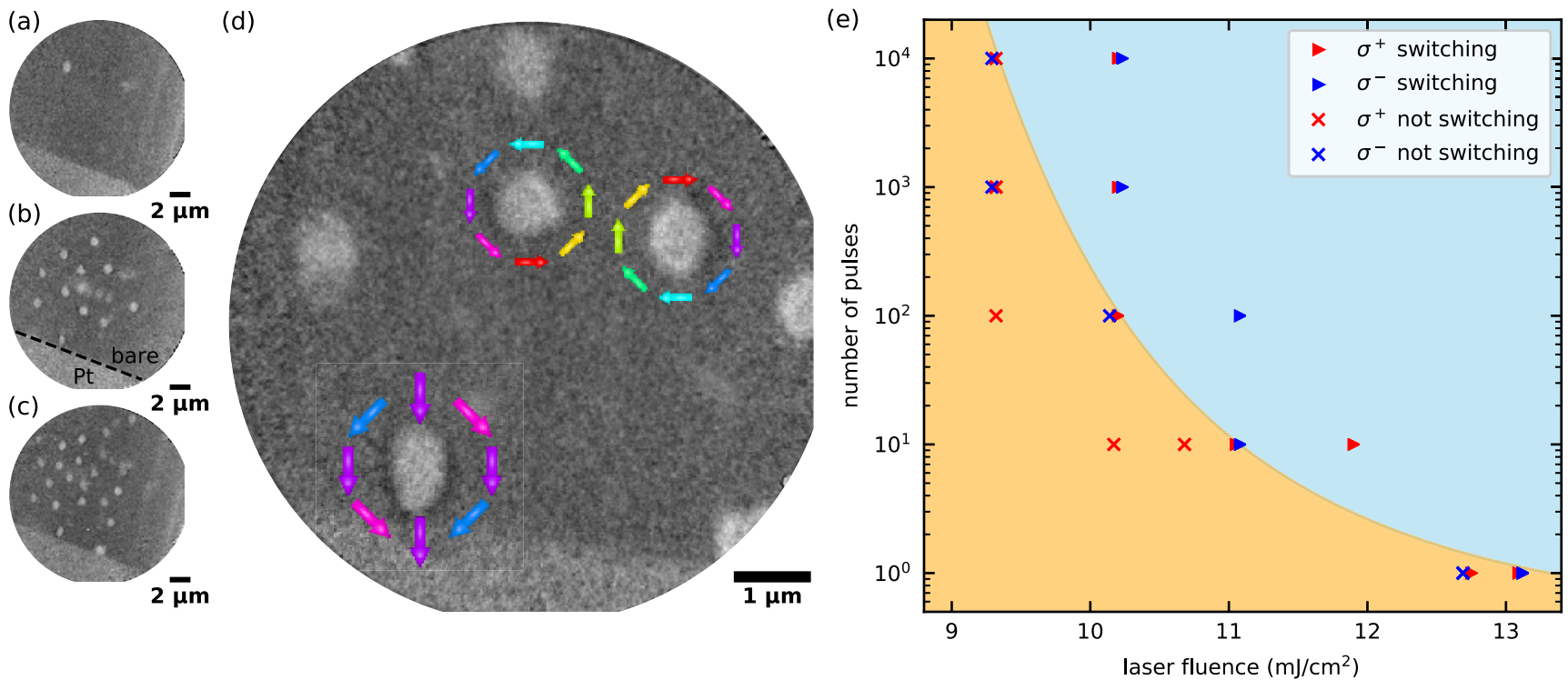

FIG. 4. Laser-induced bubble nucleation in $26.5 \mathrm{~nm}$ TmIG. (a)-(c) PEEM images of domain state in a purely out-of-plane bias field of $2.1 \mathrm{mT}$, in the initial state (a), after one laser pulse (b) and after a second laser pulse (c). Light (dark) contrast corresponds to out-of-plane (into-the-plane) magnetization. Panel (d) shows a higher-magnification image of several bubbles in (c), where the light/dark contrast at the bubble perimeter is due to the in-plane orientation of the magnetization, marked as colored arrows. (e) Laser-induced bubble nucleation thresholds versus laser fluence and pulse number. Blue and tan regions indicate presence or absence of bubble nucleation for positive and negative laser helicity. The x-ray direction in all images was top-to-bottom and approximately perpendicular to the Pt strip.

imaged using photoemission electron microscopy (PEEM) with XMCD contrast. Images were recorded at grazing incidence $\left(16^{\circ}\right.$ with respect to the surface plane) such that both the in-plane and out-of-plane magnetization orientations can be determined. Charging was avoided by covering almost the entire sample with 50-nm-thick Pt, leaving only small $10 \mu \mathrm{m}$ to $20 \mu \mathrm{m}$ wide trenches of bare film for imaging.

Figures 4(a)-4(c) show images after first saturating the sample and subsequently reducing the applied field to $B_{z}=$ $2.1 \mathrm{mT}$. At this field, the sample is expected to remain in a single-domain state, based on the hysteresis loop in Fig. 1(b). This is confirmed by the PEEM image of the initial state in Fig. 4(a). Figures 4(b) and 4(c) show PEEM images after a single laser pulse excitation [Fig. 4(b)], and after a second laser pulse excitation [Fig. 4(c)], at a laser fluence of $31 \mathrm{~mJ} / \mathrm{cm}^{2}$, respectively. We observe similar bubble domain nucleation as was observed for electrical current pulse excitation, even though the laser excitations are six orders of magnitude shorter in duration. Laser-induced bubble nucleation is progressive, with the density of bubbles increasing with increasing pulse number. The fluence threshold for bubble nucleation is not sharp, though as the fluence is reduced, the number of pulses required to nucleate bubbles increases exponentially, as seen in Fig. 4(e). The switching threshold does not depend on the helicity of the laser pulses within our experimental resolution. These results suggest that the observed laser-induced bubble nucleation is a thermal effect, similar to that observed by current injection.

The bubble chirality was directly determined using the inplane sensitivity of grazing incidence PEEM [16], as depicted in Fig. 4(d). The bubble domain walls are generally Bloch type with a random sense of rotation (clockwise or counterclock- wise). Some bubbles exhibit a mixed chirality (clockwise Bloch on one side and counterclockwise Bloch on the other side), which indicates the presence of vertical Bloch lines even though these cannot be resolved directly. The presence of Bloch lines means that some bubbles have topological charges other than unity, which distinguishes them from skyrmions in high DMI materials, where the chirality is fixed and the topological charge is always unity [14-16,35,37-39]. Interfacial DMI leading to stabilization of Néel domain walls was recently reported in ultrathin TmIG films [6,7]; the results in our thicker films suggest that the DMI effective field, which decreases with increasing film thickness, is not sufficient to overcome the magnetostatic fields that favor Bloch domain walls. The presence of Bloch lines is still surprising because the magnetic field was applied precisely in the out-of-plane direction by design. Under these conditions, bubbles in achiral hexaferrite and $\mathrm{Gd} / \mathrm{Fe}$ thin films were reported to be of random chirality but common topology $[40,41]$. We therefore conclude that DMI plays a negligible role in the bubble nucleation and stability in these samples and that Bloch lines are of sufficiently low energy to exist in these bubbles even without an in-plane field.

To further examine the effect of temperature in our sample, we imaged the domain state as the temperature was slowly increased from $T=300 \mathrm{~K}$ to $T=340 \mathrm{~K}$ in the sample cryostat, as shown in Figs. 5(b)-5(e). The sample was first saturated in a field of $B_{z}=5.3 \mathrm{mT}$ and then the field was reduced to $B_{z}=2.3 \mathrm{mT}$, reproducing the field sequence where bubbles were successfully nucleated all-optically. At $T=300 \mathrm{~K}$, the out-of-plane hysteresis loop in Fig. 5(a) shows that a saturated state is expected under these conditions, as is verified by the PEEM image in Fig. 5(b). The hysteresis 
(a)

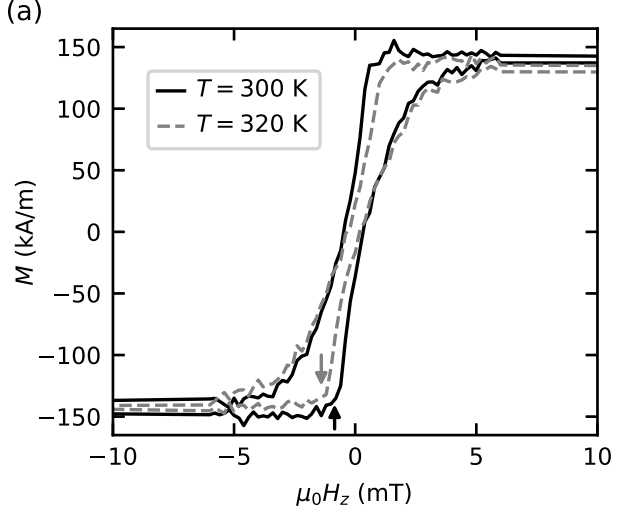

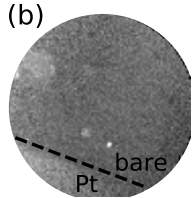

(d)

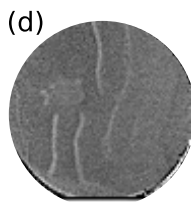

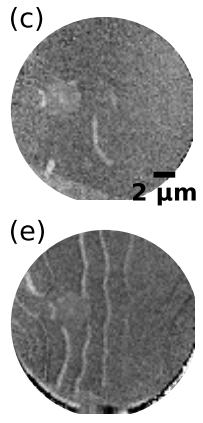

(f)

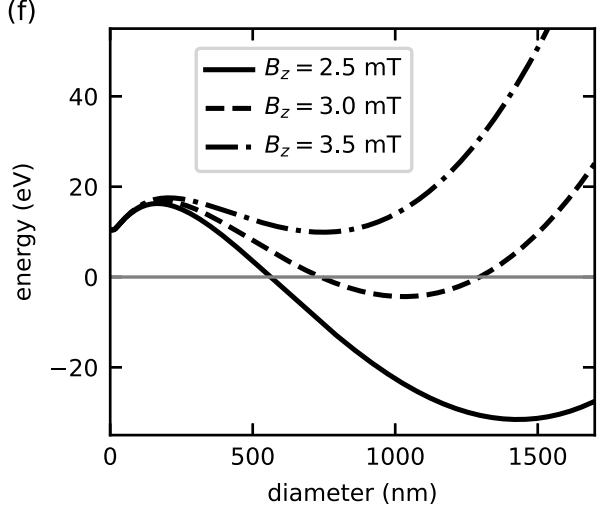

FIG. 5. Domain nucleation by quasistatic heating in $26.5 \mathrm{~nm}$ TmIG. (a) Out-of-plane hysteresis loops at temperature $T=300 \mathrm{~K}$ and $T=320 \mathrm{~K}$. The fields at which domain nucleation occurs on the increasing branch of the hysteresis loops are indicated by arrows. (b) PEEM image at $T=300 \mathrm{~K}$ after saturating the film and reducing the field to $B_{z}=2.3 \mathrm{mT}$. (c)-(e) PEEM images at temperatures of $320 \mathrm{~K}$ (c), $330 \mathrm{~K}$ (d), and $340 \mathrm{~K}$ (e). $T$ was slowly increased $(\sim 1 \mathrm{~K} / \mathrm{s})$ and the purely out-of-plane field was kept constant at $B_{z}=2.3 \mathrm{mT}$. (f) Calculated bubble energy as a function of its diameter in our TmIG material for three field values, as indicated. See Methods for parameters.

loop slightly deviates from the bare film loop in Fig. 1(b) due to pinning induced by the patterning processes, leading to a finite coercivity. Also, note that the single bubble domain in Fig. 5(b) was nucleated during a previous laser exposure and appeared to be stable even at the largest available magnetic field during our PEEM measurements (5.3 mT) [42]. Bubble stability beyond the apparent saturation field is common in perpendicular magnetic anisotropy materials [43]. At elevated temperatures, the minimum field $B_{n}$ required to maintain a uniformly magnetized or low bubble density state increases, as seen in the hysteresis loop at $T=320 \mathrm{~K}$ in Fig. 5(a). PEEM imaging in Figs. 5(b)-5(e) shows that the sample spontaneously demagnetizes as the temperature increases under constant $B_{z}$, with the density of domains increasing with increasing temperature. This result agrees with previous reports showing that the net perpendicular anisotropy decreases with increasing temperature [9], making a multidomain state more favorable. Hence, increased temperature can drive domain nucleation in these films. Bubble domains do not appear during this slow heating process.

\section{DISCUSSION}

The mechanism of fast thermal bubble nucleation in ultrathin garnet materials is different from traditional all-optical switching [44-46], all-optical topological switching [47,48], and from previously studied light-induced switching in garnet materials [49]. All these mechanisms are deterministic and involve some form of ultrafast transient phase transition. By contrast, thermal bubble nucleation is probabilistic, progressive, and can be much slower than conventional optical reversal processes. Thermal bubble nucleation can also be distinguished from helicity-dependent all-optical switching [50-54] because the helicity appears to play no role, even after thousands of pulses [Fig. 4(e)]. Moreover, the physics of switching appears to be the same regardless of whether the heat pulse is delivered by light or electrical current and insensitive to the presence of a Pt top layer.

Our measurements suggest that bubble nucleation is mediated by a transient thermal excitation over the nucleation energy barrier. As was shown in Ref. [24], the energetics of the possible multidomain morphological states (labyrinth, stripe, and bubble array) depend on the applied magnetic field, with the bubble array state becoming the ground state at higher $B_{z}$. Figure 5(f) shows the energy landscape of an isolated bubble domain as calculated using the model of Ref. [35] and the parameters of our material (see Methods). Bubble diameters are of the right order of magnitude, with small discrepancies to the observed bubble sizes likely originating from higher order anisotropy terms not included in our model. The energy barriers exceed several hundred times the thermal energy at room temperature $(26 \mathrm{meV})$. Therefore, morphological transitions between metastable and stable states do not readily occur during quasistatic variation of the field or temperature. Instead, the experimental results presented here suggest that fast thermal excitations, delivered by Joule heat pulses during current injection or ultrafast laser pulses, can drive the system over these energy barriers to a bubble ground state configuration.

\section{CONCLUSIONS}

In summary, we have successfully prepared sub-30-nmthick, sheared loop, epitaxial thulium iron garnet films and demonstrated a process to back-thin their single-crystalline substrates down to soft $\mathrm{x}$-ray transparent thicknesses without changing the strain-induced magnetic properties of the films. We found that submicrometer sized bubble domains are readily nucleated in these films by single heat pulses, where the excitation can be as short as $80 \mathrm{fs}$ using an ultrafast laser. Our results suggest a strategy to nucleate magnetic bubble domains in insulating magnetic garnet films and demonstrate how $\mathrm{x}$ ray imaging can be applied to study the resulting magnetic textures statically and upon in situ excitation. Although the relatively thick films in the present study show negligible Dzyaloshinskii-Moriya interactions, our results suggest that in ultrathin rare earth iron garnet films, in which interfacial DMI has recently been found, fast thermal excitations might be used to controllably nucleate chiral magnetic skyrmions. 


\section{METHODS}

TmIG films were deposited using pulsed laser deposition (PLD) on single-crystal (111) GGG substrates as described in Ref. [55]. The PLD used a $248 \mathrm{~nm}$ wavelength $\mathrm{KrF}$ excimer laser with $10 \mathrm{~Hz}$ repetition rate and a heated substrate stage. The target used was a commercially available TmIG target with a $99.9 \%$ elemental purity. Pt tracks were prepared by sputtering and patterned by direct laser optical lithography and liftoff. Oxygen plasma cleaning was employed to remove resist residues from the TmIG surface before Pt deposition. Contact pads were subsequently prepared in a similar manner. Thinning of the substrates from the back side was performed as a last step using mechanical polishing followed by focused ion beam milling. Alignment with the front side textures was performed by first etching markers with the FIB on the back side and then checking the position of the Pt tracks with respect to those markers via optical microscopy through the transparent sample.

Laser pulses were generated by a Femtolasers Scientific XL Ti:sapphire oscillator with a central wavelength of $800 \mathrm{~nm}$ and a pulse duration of 80 fs (full width at half maximum, FWHM). The spot size on the TmIG surface was $(4.3 \pm$ $0.1 \mu \mathrm{m}) \times(6.3 \pm 0.2) \mu \mathrm{m}$. The spot size and fluences were calibrated as described in Ref. [56]. During all measurements in Fig. 4(e), the temperature was kept constant at $299.5 \pm$ $0.5) \mathrm{K}$.

The analytical model in Fig. 5(f) is based on Ref. [35] using a film thickness of $26.5 \mathrm{~nm}$, a saturation magnetization of $M_{s}=140 \mathrm{kA} / \mathrm{m}$, and an anisotropy field of $H_{k}=80 \mathrm{kA} / \mathrm{m} \quad\left(B_{k}=100 \mathrm{mT}\right)$ as determined from the in-plane loop in Fig. 1(b), an exchange constant of $A=2.3 \mathrm{pJ} / \mathrm{m}$ [57], and zero DMI.

\section{ACKNOWLEDGMENTS}

We thank Ulrike Eigenthaler, Thomas Meisner, and Kim Kisslinger for their extensive support in preparing the membrane samples. This work was supported by the US Defense Advanced Research Projects Agency (DARPA) under Project No. HR0011834375, NSF Grant No. DMR1808190, and by the SMART Center sponsored by NIST and SRC. Part of the measurements were carried out at the beamlines UE46 (MAXYMUS) and UE49 (SPEEM) at Helmholtz-Zentrum Berlin. We thank HZB for the allocation of synchrotron radiation beamtime. We acknowledge the Paul Scherrer Institut, Villigen, Switzerland, for provision of synchrotron radiation beamtime at beamlines X07DA (PolLux) and X11MA (SIM) of the SLS. This research used resources of the Center for Functional Nanomaterials, which is a US DOE Office of Science Facility, at Brookhaven National Laboratory under Contract No. DE-SC0012704. L.C. acknowledges financial support from the National Science Foundation Graduate Research Fellowship Program and the National GEM Consortium.

F.B. and M.A.M. contributed equally to this work.
[1] A. H. Bobeck and E. Della Torre, Magnetic Bubbles (NorthHolland Publishing Company, Amsterdam, 1975).

[2] A. P. Malozemoff and J. C. Slonczewski, Magnetic Domain Walls in Bubble Materials (Academic Press, New York, 1979).

[3] Edited by H. Jouve, Magnetic Bubbles (Academic Press, London, 1986).

[4] H. Chang, P. Li, W. Zhang, T. Liu, A. Hoffmann, L. Deng, and M. Wu, IEEE Magn. Lett. 5, 1 (2014).

[5] L. J. Cornelissen, J. Liu, R. A. Duine, J. B. Youssef, and B. J. van Wees, Nat. Phys. 11, 1022 (2015).

[6] C. O. Avci, E. Rosenberg, L. Caretta, F. Büttner, M. Mann, C. Marcus, D. Bono, C. A. Ross, and G. S. D. Beach, Nat. Nanotechnol. 14, 561 (2019).

[7] S. Vélez, J. Schaab, M. S. Wörnle, M. Müller, E. Gradauskaite, P. Welter, C. Gutgsell, C. Nistor, C. L. Degen, M. Trassin, M. Fiebig, and P. Gambardella, Nat. Commun. 10, 4750 (2019).

[8] M. Kubota, A. Tsukazaki, F. Kagawa, K. Shibuya, Y. Tokunaga, M. Kawasaki, and Y. Tokura, Appl. Phys. Express 5, 103002 (2012).

[9] A. Quindeau, C. O. Avci, W. Liu, C. Sun, M. Mann, A. S. Tang, M. C. Onbasli, D. Bono, P. M. Voyles, Y. Xu, J. Robinson, G. S. D. Beach, and C. A. Ross, Adv. Electron. Mater. 3, 1600376 (2017).

[10] C. O. Avci, A. Quindeau, C.-F. Pai, M. Mann, L. Caretta, A. S. Tang, M. C. Onbasli, C. A. Ross, and G. S. D. Beach, Nat. Mater. 16, 309 (2016).

[11] Q. Shao, Y. Liu, G. Yu, S. K. Kim, X. Che, C. Tang, Q. L. He, Y. Tserkovnyak, J. Shi, and K. L. Wang, Nat. Electron. 2, 182 (2019).
[12] A. S. Ahmed, A. J. Lee, N. Bagués, B. A. McCullian, A. M. A. Thabt, A. Perrine, P.-K. Wu, J. R. Rowland, M. Randeria, P. C. Hammel, D. W. McComb, and F. Yang, Nano Lett. 19, 5683 (2019).

[13] F. Büttner, C. Moutafis, M. Schneider, B. Krüger, C. M. Günther, J. Geilhufe, C. von Korff Schmising, J. Mohanty, B. Pfau, S. Schaffert, A. Bisig, M. Foerster, T. Schulz, C. a. F. Vaz, J. H. Franken, H. J. M. Swagten, M. Kläui, and S. Eisebitt, Nat. Phys. 11, 225 (2015).

[14] S. Woo, K. Litzius, B. Krüger, M.-Y. Im, L. Caretta, K. Richter, M. Mann, A. Krone, R. M. Reeve, M. Weigand, P. Agrawal, I. Lemesh, M.-A. Mawass, P. Fischer, M. Kläui, and G. S. D. Beach, Nat. Mater. 15, 501 (2016).

[15] C. Moreau-Luchaire, C. Moutafis, N. Reyren, J. Sampaio, C. A. F. Vaz, N. V. Horne, K. Bouzehouane, K. Garcia, C. Deranlot, P. Warnicke, P. Wohlhüter, J.-M. George, M. Weigand, J. Raabe, V. Cros, and A. Fert, Nat. Nanotechnol. 11, 444 (2016).

[16] O. Boulle, J. Vogel, H. Yang, S. Pizzini, D. de Souza Chaves, A. Locatelli, T. O. Menteş, A. Sala, L. D. Buda-Prejbeanu, O. Klein, M. Belmeguenai, Y. Roussigné, A. Stashkevich, S. M. Chérif, L. Aballe, M. Foerster, M. Chshiev, S. Auffret, I. M. Miron, and G. Gaudin, Nat. Nanotechnol. 11, 449 (2016).

[17] K. Litzius, I. Lemesh, B. Krüger, P. Bassirian, L. Caretta, K. Richter, F. Büttner, K. Sato, O. A. Tretiakov, J. Förster, R. M. Reeve, M. Weigand, I. Bykova, H. Stoll, G. Schütz, G. S. D. Beach, and M. Kläui, Nat. Phys. 13, 170 (2017).

[18] A. Soumyanarayanan, M. Raju, A. L. Gonzalez Oyarce, A. K. C. Tan, M.-Y. Im, A. P. Petrović, P. Ho, K. H. Khoo, 
M. Tran, C. K. Gan, F. Ernult, and C. Panagopoulos, Nat. Mater. 16, 898 (2017).

[19] K. Zeissler, S. Finizio, K. Shahbazi, J. Massey, F. A. Ma’Mari, D. M. Bracher, A. Kleibert, M. C. Rosamond, E. H. Linfield, T. A. Moore, J. Raabe, G. Burnell, and C. H. Marrows, Nat. Nanotechnol. 13, 1161 (2018).

[20] L. Caretta, M. Mann, F. Büttner, K. Ueda, B. Pfau, C. M. Günther, P. Hessing, A. Churikova, C. Klose, M. Schneider, D. Engel, C. Marcus, D. Bono, K. Bagschik, S. Eisebitt, and G. S. D. Beach, Nat. Nanotechnol. 13, 1154 (2018).

[21] F. Sayetat, J. Magn. Magn. Mater. 58, 334 (1986).

[22] C. Kooy and U. Enz, Philips Res. Rep. 15, 7 (1960).

[23] J. A. Cape and G. W. Lehman, J. Appl. Phys. 42, 5732 (1971).

[24] I. Lemesh, K. Litzius, M. Böttcher, P. Bassirian, N. Kerber, D. Heinze, J. Zázvorka, F. Büttner, L. Caretta, M. Mann, M. Weigand, S. Finizio, J. Raabe, M.-Y. Im, H. Stoll, G. Schütz, B. Dupé, M. Kläui, and G. S. D. Beach, Adv. Mater. 30, 1805461 (2018).

[25] G. Schütz, W. Wagner, W. Wilhelm, P. Kienle, R. Zeller, R. Frahm, and G. Materlik, Phys. Rev. Lett. 58, 737 (1987).

[26] A. A. Thiele, J. Appl. Phys. 41, 1139 (1970).

[27] F. Büttner, I. Lemesh, M. Schneider, B. Pfau, C. M. Günther, P. Hessing, J. Geilhufe, L. Caretta, D. Engel, B. Krüger, J. Viefhaus, S. Eisebitt, and G. S. D. Beach, Nat. Nanotechnol. 12, 1040 (2017).

[28] W. Legrand, D. Maccariello, N. Reyren, K. Garcia, C. Moutafis, C. Moreau-Luchaire, S. Collin, K. Bouzehouane, V. Cros, and A. Fert, Nano Lett. 17, 2703 (2017).

[29] A. Hrabec, J. Sampaio, M. Belmeguenai, I. Gross, R. Weil, S. M. Chérif, A. Stashkevich, V. Jacques, A. Thiaville, and S. Rohart, Nat. Commun. 8, 15765 (2017).

[30] I. Lemesh, F. Büttner, and G. S. D. Beach, Phys. Rev. B 95, 174423 (2017).

[31] A. Brataas and K. M. D. Hals, Nat. Nanotechnol. 9, 86 (2014).

[32] F. Hellman, A. Hoffmann, Y. Tserkovnyak, G. S. D. Beach, E. E. Fullerton, C. Leighton, A. H. MacDonald, D. C. Ralph, D. A. Arena, H. A. Dürr, P. Fischer, J. Grollier, J. P. Heremans, T. Jungwirth, A. V. Kimel, B. Koopmans, I. N. Krivorotov, S. J. May, A. K. Petford-Long, J. M. Rondinelli, N. Samarth, I. K. Schuller, A. N. Slavin, M. D. Stiles, O. Tchernyshyov, A. Thiaville, and B. L. Zink, Rev. Mod. Phys. 89, 025006 (2017).

[33] A. A. Thiele, Phys. Rev. Lett. 30, 230 (1973).

[34] R. Tomasello, E. Martinez, R. Zivieri, L. Torres, M. Carpentieri, and G. Finocchio, Sci. Rep. 4, 6784 (2014).

[35] F. Büttner, I. Lemesh, and G. S. D. Beach, Sci. Rep. 8, 4464 (2018).

[36] L. Gierster, L. Pape, A. A. Ünal, and F. Kronast, Rev. Sci. Instrum. 86, 023702 (2015).

[37] S. Mühlbauer, B. Binz, F. Jonietz, C. Pfleiderer, A. Rosch, A. Neubauer, R. Georgii, and P. Böni, Science 323, 915 (2009).

[38] X. Z. Yu, Y. Onose, N. Kanazawa, J. H. Park, J. H. Han, Y. Matsui, N. Nagaosa, and Y. Tokura, Nature (London) 465, 901 (2010).

[39] W. Jiang, P. Upadhyaya, W. Zhang, G. Yu, M. B. Jungfleisch, F. Y. Fradin, J. E. Pearson, Y. Tserkovnyak, K. L. Wang, O. Heinonen, S. G. E. t. Velthuis, and A. Hoffmann, Science 349, 283 (2015).
[40] X. Yu, M. Mostovoy, Y. Tokunaga, W. Zhang, K. Kimoto, Y. Matsui, Y. Kaneko, N. Nagaosa, and Y. Tokura, Proc. Natl. Acad. Sci. USA 109, 8856 (2012).

[41] S. A. Montoya, S. Couture, J. J. Chess, J. C. T. Lee, N. Kent, D. Henze, S. K. Sinha, M.-Y. Im, S. D. Kevan, P. Fischer, B. J. McMorran, V. Lomakin, S. Roy, and E. E. Fullerton, Phys. Rev. B 95, 024415 (2017).

[42] L. Gierster, A. A. Ünal, L. Pape, F. Radu, and F. Kronast, Ultramicroscopy, 159, 508 (2015), Special Issue: LEEM-PEEM 9.

[43] J. E. Davies, O. Hellwig, E. E. Fullerton, G. Denbeaux, J. B. Kortright, and K. Liu, Phys. Rev. B 70, 224434 (2004).

[44] C. D. Stanciu, F. Hansteen, A. V. Kimel, A. Kirilyuk, A. Tsukamoto, A. Itoh, and T. Rasing, Phys. Rev. Lett. 99, 047601 (2007).

[45] I. Radu, K. Vahaplar, C. Stamm, T. Kachel, N. Pontius, H. A. Dürr, T. A. Ostler, J. Barker, R. F. L. Evans, R. W. Chantrell, A. Tsukamoto, A. Itoh, A. Kirilyuk, T. Rasing, and A. V. Kimel, Nature (London) 472, 205 (2011).

[46] T. A. Ostler, J. Barker, R. F. L. Evans, R. W. Chantrell, U. Atxitia, O. Chubykalo-Fesenko, S. El Moussaoui, L. Le Guyader, E. Mengotti, L. J. Heyderman, F. Nolting, A. Tsukamoto, A. Itoh, D. Afanasiev, B. A. Ivanov, A. M. Kalashnikova, K. Vahaplar, J. Mentink, A. Kirilyuk, T. Rasing, and A. V. Kimel, Nat. Commun. 3, 666 (2012).

[47] G. Berruto, I. Madan, Y. Murooka, G. M. Vanacore, E. Pomarico, J. Rajeswari, R. Lamb, P. Huang, A. J. Kruchkov, Y. Togawa, T. LaGrange, D. McGrouther, H. M. Rønnow, and F. Carbone, Phys. Rev. Lett. 120, 117201 (2018).

[48] S.-G. Je, P. Vallobra, T. Srivastava, J.-C. Rojas-Sánchez, T. H. Pham, M. Hehn, G. Malinowski, C. Baraduc, S. Auffret, G. Gaudin, S. Mangin, H. Béa, and O. Boulle, Nano Lett. 18, 7362 (2018).

[49] A. Stupakiewicz, K. Szerenos, D. Afanasiev, A. Kirilyuk, and A. V. Kimel, Nature (London) 542, 71 (2017).

[50] C.-H. Lambert, S. Mangin, B. S. D. C. S. Varaprasad, Y. K. Takahashi, M. Hehn, M. Cinchetti, G. Malinowski, K. Hono, Y. Fainman, M. Aeschlimann, and E. E. Fullerton, Science 345, 1337 (2014).

[51] M. S. El Hadri, P. Pirro, C.-H. Lambert, S. Petit-Watelot, Y. Quessab, M. Hehn, F. Montaigne, G. Malinowski, and S. Mangin, Phys. Rev. B 94, 064412 (2016).

[52] J. Gorchon, Y. Yang, and J. Bokor, Phys. Rev. B 94, 020409(R) (2016).

[53] R. Medapalli, D. Afanasiev, D. K. Kim, Y. Quessab, S. Manna, S. A. Montoya, A. Kirilyuk, T. Rasing, A. V. Kimel, and E. E. Fullerton, Phys. Rev. B 96, 224421 (2017).

[54] R. John, M. Berritta, D. Hinzke, C. Müller, T. Santos, H Ulrichs, P. Nieves, J. Walowski, R. Mondal, O. ChubykaloFesenko, J. McCord, P. M. Oppeneer, U. Nowak, and M. Münzenberg, Sci. Rep. 7, 4114 (2017).

[55] E. R. Rosenberg, L. Beran, C. O. Avci, C. Zeledon, B. Song, C. Gonzalez-Fuentes, J. Mendil, P. Gambardella, M. Veis, C. Garcia, G. S. D. Beach, and C. A. Ross, Phys. Rev. Materials 2, 094405 (2018)

[56] A. Arora, M.-A. Mawass, O. Sandig, C. Luo, A. A. Ünal, F. Radu, S. Valencia, and F. Kronast, Sci. Rep. 7, 9456 (2017).

[57] S. Ding, A. Ross, R. Lebrun, S. Becker, K. Lee, I. Boventer, S. Das, Y. Kurokawa, S. Gupta, J. Yang, G. Jakob, and M. Kläui, Phys. Rev. B 100, 100406 (2019). 